\title{
An Adaptive Weighted Image Denoising Method Based on Morphology
}

\author{
Jinjuan Wang \\ Hunan University of International Economics, Changsha 410205, Hunan, China \\ Shan Duan \\ Hunan University of International Economics, Changsha 410205, Hunan, China \\ Qun Zhou \\ Hunan University of International Economics, Changsha 410205, Hunan, China
}

Received: August 17, 2020. Revised: March 22, 2021. Accepted: April 2, 2021. Published: April 8, 2021.

\begin{abstract}
In its generation, transmission and record, image signal is often interfered by various noises, which have severally affected the visual effects of images; therefore, it is a very important pre-processing step to take proper approaches to reduce noises. Conventional denoising methods have also blurred image edge information while removing noises, which can be overcome by the method based on mathematical morphology. While eliminating different noises from images, it can not only keep clear object edges, but also preserve as many image details as possible and it also has excellent capacities in noise resistance and edge preservation. With image denoising and mathematical morphology as the research subject, this paper analyzes the generation and characteristics of common image noises, studies the basic theories of mathematical morphology and its applications in image processing, discusses the method to select structural elements in mathematical morphology and proposes a filtering algorithm which combines image denoising and mathematical morphology. This method conducts morphological filtering and denoising on noised image with filter cascade and its performance is verified with stimulation testing. The experiment results prove that the approach to build the morphological filter into cascaded filter through series and parallel connection can to a certain extent, affect the effect of common filter while being applied to different image processing.
\end{abstract}

Keywords-Image Denoising; Mathematical Morphology; Adaptive Weight; Image Details;

\section{INTRODUCTION}

$\mathrm{T}$ he growth and popularization of computer technology has brought wide applications of digital images. However, in the shooting, transmitting and processing of images, a large number of noises have been added to images due to the restrictions of the devices used and the transmission channels. These noises have been so severe that they have influenced the visual effects of images and even hindered peoples' normal recognition[1]. Mathematical morphology is such a discipline built upon strict theoretical foundation of mathematics and its basic ideas and methods have played a major role in the theories and techniques of image processing[2]. In fact, a new image processing method and theory has been constituted and even become an important research field in computer digital image processing. Some detail information of noises and image shares some similarities, which has further enhanced the image fuzziness; worst still, human vision is blur and difficult to tell the grayscale from black to white. Such uncertainty and inaccuracy are mainly reflected in the uncertainty of image grayscale[3]. The uncertainty of geometrical shapes and the knowledge of uncertainty are difficult to be solved by classic mathematical theories and this uncertainty is not all random and so it is also difficult to be settled through probability theory. To obtain high-quality digital images, researchers have been exploring various effective image denoising methods. Extract useful information from the image, improve its signal-to-noise ratio and resolution ratio and improve image quality to facilitate higher-level processing. In this process, to denoise image is an essential link. Humans are the final observer of image processing; therefore, in image processing and recognition, image features and human visual characteristics must be taken into full consideration. The imaging process is a many-to-one mapping process, from which point of view, it has been decided that image has many uncertainties and inaccuracy, i.e. fuzziness[4]. Mathematical morphology can simplify image 
data, preserve its basic shape property, remove irrelevant structures and collect image information with a "probe" called structural element. When the probe keeps moving in the image, it can observe and explore the interrelation among different parts to understand its structural features[5]. This paper mainly use the method based on mathematical morphology to make systematic and in-depth research on image denoising.

Mathematical morphology is a subject which was built by French and German scientists in their study of rock structure. Morphology is used to obtain the topological and structural information of objects and it can get more essential form of object through some operations on the correlation of objects and structural elements[6]. In general, image denoising technology can be divided into 2 types: spatial-domain denoising technology and transform-domain denoising technology. Targeted on the image plane, the former directly processes image pixels. It first determines whether a pixel is a noise point. If yes, it gives a new value to it; if not, its original value is its output. The latter doesn't rely on the recognition and removal of noise points; instead, it is a mean method that makes average computation on the entire image[7]. In recent years, with the gradual deepening theories and applications of different mathematical branches, mathematical morphology, fuzzy mathematics, genetic algorithm, wavelet theory and information theory etc have made great progress in the applications of image denoising technology and many new denoising algorithms have come into being, including those based on mathematical morphology, fuzzy theory, neural networks, and wavelet theory. Their applications in image processing mainly includes to use the basic operations of morphology to observe and process the image so as to improve its quality; and to describe and define the geometrical parameters and features of the image, including area, perimeter, connectivity, granularity, framework and directivity. Linear filter and non-linear filter are the most common-used spatial-domain denoising filter at present. Mean filter and linearly weighted filter belong to linear filter while median filter and morphological filter are non-linear filter[8] [9].

The specific contributions of this paper include the following:

1) Based on the theory of mathematical morphology, this paper analyzes the generation and characteristics of common image noise, studies the denoising algorithm of binary image, gray image and color image, studies the basic theory of mathematical morphology and its application in image processing, and discusses the selection method of structural elements in mathematical morphology.

2) In this paper, the image denoising and mathematical morphology are taken as the research object, and the traditional morphological denoising algorithm is improved and innovated, proposed a filtering algorithm that has combined image denoising and mathematical morphology based on the selection of multi-structural elements in morphology.

3) The experiment result has shown that in both visual effects and PSNR value, our proposed algorithm can make good results and it has led to impressive denoising effects especially for the images with rich texture details.
With image denoising as the research object, this paper has first analyzed the characteristics and types of image noises, studied the noise models and summarized the common image denoising algorithms. Then it has analyzed the principles and characteristics of mathematical morphology and proposed an adaptive weighted multi-structural morphology denoising method. After that, it has introduced the design of filter and explained how to make series and parallel connection on filters of different shapes and dimensions to build cascaded filter. Finally, it has made simulation experiment on this method.

\section{IMAGE DENOISING}

Noise refers to the factors which affect the understanding of information from various information sources through sense organs; from this perspective, when conveying information to the receiver from the shooting object or information source with certain method, if 2D brightness distribution is to be received through vision, then the brightness distribution that interrupts such reception is called image noise, which is usually quantized or described with signal-to-noise ratio (SNR) [10]. For example, for a black-and-white image, assume its plane brightness distribution is $f(x, y)$, and then the brightness distribution $R(x, y)$ which interferes its reception can be called as image noise ${ }^{[2]}$. But theoretically, noise can be defined as "unpredictable random error which can only be recognized by probabilistic method". So it is proper to see image noise as a multi-dimensional random process and the method to describe noise can totally use the description of random process, i.e. the probability distribution function and probability density distribution function. In practical applications, its numerical characteristics such as mean variance and relevant functions are usually used as they can reflect the features of noise from some aspects[11].

\section{A. Characteristics of Image Noises}

Image noises can blur the image and even overwhelm image features, bringing difficulty to image analysis. Image noises usually have the following characteristics[12]:

(1) Noises are irregular in the image with regards to distribution and size, namely that it has randomness.

(2) Noises are usually correlated with the image. For instance, camera signal is correlated with noises: the dark part has loud noises and the bright part low noises. There are definitely quantizing noises in digital image processing technology and it has something to do with the image phase. For example, when the image content is close to flat, the quantizing noise shows false contour, but the random noises in the image signal will make quantizing noise less obvious because of microphonic effect.

(3) Noises are superimposed. In cascade image transmission system, the noises in each part can have their power added if they are of the same kind and the SNR will drop successively. If not the same type, they should be treated differently and the impact of visually measuring characteristics shall be taken into consideration. 


\section{B. Several Common Image Noises}

(1)Additive noise and multiplicative noise

According to the relationship between noises and signals, image noise can be divided into additive noise and multiplicative noise. To facilitate analysis and processing, multiplicative noise is usually seen as additive noise and signal is often independently from noises.

Assume that the signal is $S(t)$ and the noise is $n(t)$, if the mixed overlayed waveform is $S(t)+n(t)$, it is called as additive noise. Additive noise is irrelevant to the strength of image signal. If the overlayed waveform is $S(t)[1+n(t)]$, it is called as multiplicative noise. This type of noise is related to signal strength and it usually varies with image signal, such as the noise in the flying-spot scanning image, TV scanning raster and filter grain[13].

(2)External noise and internal noise

According to the generation reasons, image noise includes external noise and internal noise. External noise refers to the noises caused by external disturbance entering the system in the form of electromagnetic wave or via power supply, including the electromagnetic interference generated by external electrical equipment and impulse interference from the discharge of celestial body. The noises caused by the internal electrical equipment is internal noise, such as the mutual interference of internal circuit. Generally, internal noises have the following 4 types: (1) the noises caused by the basic properties of light and electricity; (2) the noises generated by the mechanical motion of electric appliance; (3) the noises caused by the equipment materials; and (4) the noises caused by the circuit of internal equipment[14].

(3) Stationary noise and non-stationary noise

According to statistical property, image noise can be divided into stationary noise and non-stationary noise. Those whose statistical property doesn't change over time are stationary noises and those whose statistical property changes over time are non-stationary noises.

(4) Several other noises

Quantizing noise is the main noise source of digital image and its magnitude shows the difference between digital image and the original image. The best way to reduce such noise is to select the o-level optimization measure by adopting grayscale probability density function. "Salt and pepper" noise: this kind of noise means the white dots in black images and black dots in white images caused by image incision as well as the converter noise caused by inverse image transformation after introducing error in transform domain[15].

As defined by the shape of how noise magnitude is distributed over time, the noise whose magnitude distribution is Gaussian distribution is called Gaussian noise and that according to Rayleigh distribution is called Rayleigh noise. According to the shape of noise spectrum, the noise with evenly spectrum distribution is called white noise. The noise with spectrum inversely proportional to frequency is called $1 / \mathrm{f}$ noise and that with spectrum proportional to frequency square is triangle noise[16].

\section{Image Noise Model}

The noises contained in actually acquired images can be classified according to their kinds. Based on the probability distribution of noises, they can be divided into Gaussian noise, Rayleigh noise, Gamma noise, exponentially distributed noise and uniformly distributed noise.

(1)Gaussian noise

Due to its easy mathematical treatability in spatial and frequency domain, Gaussian noise is also known as normal noise. As a matter of fact, this easy treatability is very convenient, making Gaussian model frequently used in critical conditions. The PDF of Gaussian random variable $\mathrm{z}$ is given by the following formula[17]:

$$
p(z)=\frac{1}{\sqrt{2 \pi} \sigma} \exp \left[-(z-u)^{2} / 2 \sigma^{2}\right]
$$

Where $z$ represents the grayscale value, $\mu$ the mean value or expected value of $z$ and $\sigma$ the standard deviation of $z$. The square of standard deviation $\sigma^{2}$ is called the variance of $z$.

(2) Rayleigh noise

The probability density function of Rayleigh noise is shown in the following[18]:

$$
p(z)=\left\{\begin{array}{rr}
\frac{2}{b}(z-a) \exp \left[-(z-a)^{2} / b\right] & z \geq a \\
0 & z<a
\end{array}\right.
$$

The mean value and variance of probability density are provided in the following formula:

$$
\left\{\begin{array}{c}
\mu=a+\sqrt{\pi b / 4} \\
\sigma^{2}=b(4-\pi) / 4
\end{array}\right.
$$

(3) Gamma noise

The PDF of Gamma noise is defined as follows:

$$
p(z)=\left\{\begin{array}{cr}
\frac{a^{b} z^{b-1}}{(b-1) !} e^{-a x} & z \geq 0 \\
0 & z<0
\end{array}\right.
$$

Where $a>0, b$ is a positive integer and "!" means factorial. The mean and variance of its density are indicated as follows:

$$
\left\{\begin{array}{c}
\mu=\frac{b}{a} \\
\sigma^{2}=\frac{b}{a^{2}}
\end{array}\right.
$$


Though Formula (4) is often used to represent Gamma density; strictly speaking, it is true only when the denominator is Gamma function $\Gamma(b)$ and when the denominator is as shown above, the density approximation is called Irish density[19].

(4) Exponentially distributed noise

The PDF of exponent noise is offered by the following formula[20]:

$$
p(z)=\left\{\begin{array}{cc}
a z^{-a x} & z \geq 0 \\
0 & z<0
\end{array}\right.
$$

Where $a>0$. The expected value and variance of probability density function are as follows:

$$
\left\{\begin{array}{c}
\mu=\frac{1}{a} \\
\sigma^{2}=\frac{1}{a^{2}}
\end{array}\right.
$$

(5) Uniformly distributed noise

The probability density of uniformly distributed noise is given as follows[21]:

$$
p(z)=\left\{\begin{array}{cc}
\frac{1}{b-a} a \leq z \leq b \\
0 \quad \text { others }
\end{array}\right.
$$

The expected value and variance of probability density function can be sought by the following formula:

$$
\left\{\begin{array}{c}
\mu=\frac{\mathrm{a}+\mathrm{b}}{2} \\
\sigma^{2}=\frac{(b-a)^{2}}{12}
\end{array}\right.
$$

(6) Impulse noise (salt and pepper noise)

The PDF of (bipolar) impulse noise can be found according to below:

$$
p(z)=\left\{\begin{array}{c}
P a(z=a) \\
P b(z=b) \\
0(\text { others })
\end{array}\right.
$$

If $b>a$, grayscale value $\mathrm{b}$ will be shown as a bright spot in the image; otherwise, the value of a will be a dark spot. If $P a$ or $P b$ is 0 , impulse noise is called unipolar impulse. If both $P a$ and $P b$ are not zero and especially when they are approximately equal, the value of impulse noise will be similar to the pepper and salt particles randomly distributed in the image[22]. For this reason, bipolar impulse noise is also known as salt-and-pepper noise.

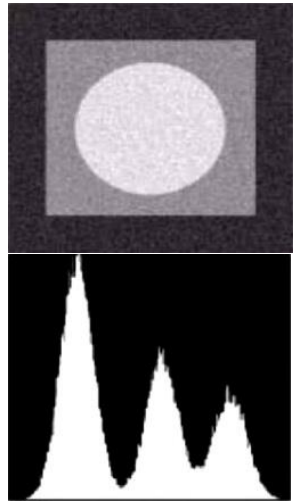

(a) Gaussian noise

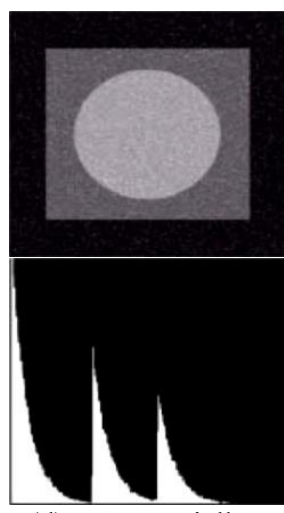

(d) Exponentially distributed noise

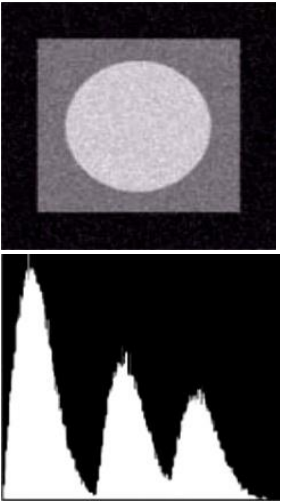

(b) Rayleigh noise

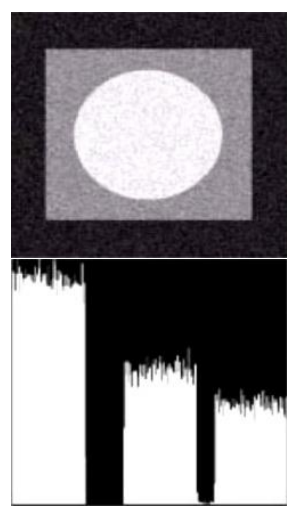

(e)Uniformly distributed noise

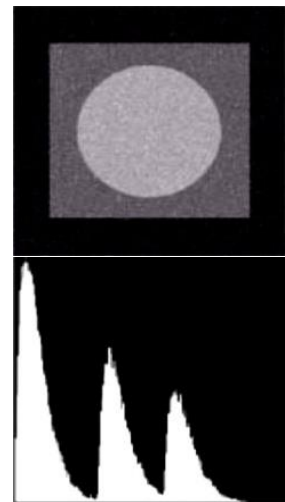

(c) Gamma noise

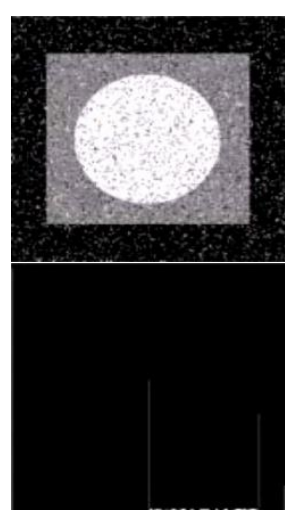

(f) Impulse noise (salt and pepper noise)
Fig.1 Different noise models and histograms

Noise impulse can be positive or negative. Calibration is usually a part of image digitization. As impulse interference is usually bigger than the strength of image signal, in an image, impulse noise is often digitized as the maximum (pure black or pure white). In this way, it is usually hypothesized that $a$ and $b$ are saturation values; from a certain sense, in digital image, they are equal to the possible maximum and minimum values. As a result, negative impulse appears in the image as a black spot. And for the same reason, positive impulse shows up in the image as white spots.

\section{Image Denoising}

Image denoising algorithms include the following types:

(1) Spatial-domain filter

Spatial filter directly conducts data operations in the original image and processes the grayscale value of pixels. The common spatial-domain image denoising algorithms include neighborhood averaging method, median filtering, and low-pass filtering etc.

(2) Transform-domain filter

Transform-domain image denoising method is to conduct certain image transformation to convert the image from the spatial domain into transform domain. Then it processes the transform coefficients in the transform domain and afterwards, it conducts inverse transform to convert the image from transform domain into spatial domain. After this process, the image noises are removed. There are many ways to transform 
the image from spatial domain to transform domain, such as Fourier transform, Walsh-hadamar transform, cosine transform, K-L transform and wavelet transform and so on. Among them, Fourier transform and wavelet transform are frequently used in image denoising[23].

(3) Partial differential equation

Partial differential equation mainly works on low-level image processing and makes good results. It is aeolotropic and when being applied in image denoising, it can remove noises and well preserve edges. The application of partial differential equation is mainly the basic iterative format and with update over time, the image gradually approximates the expected effect and the improved subsequent work. This method has a large selection room in determining diffusion coefficients and it can diffuse both forward and backward; therefore, it has the capacities to smooth the image and sharpen the edges ${ }^{[5]}$. Partial differential equation can have better effect in the image processing with a low noise density; but when handling images of high noise density, its effect is not good and it takes significantly more time.

(4) Variational method

The image denoising method through mathematics is based on the idea of variational method. It determines the energy function of the image, and minimizes the function to make the image reach a smooth state. The total variation (TV) model which has now been widely applied is this kind of method, the key to which is to find the proper energy equation so as to ensure smooth evolution and get ideal results[24].

\section{AdAPTIVE WeIGHTED MUlti-STRUCTURAL MORPHOLOGICAL DENOISING}

In grayscale morphology, the intersection and union used in binary morphology are respectively replaced by the minimum and maximum. The operands here are not seen as a set, but an image function. In the following text, it is hypothesized that $f(x, y)$ represents the input image and $b(x, y)$ refers to the structural element, which is also a sub-image.

1) Grayscale corrosion

Perform grayscale corrosion to the input image $f$ with structural element $b$ and mark as $f \Theta b$. Its definition is as follows:

$$
\begin{aligned}
& (f \Theta b)(s, t)=\min \{f(s+x, t+y)-b(x, y) \mid \\
& \left.(s+x, t+y) \in D_{f},(x, y) \in D_{b}\right\}
\end{aligned}
$$

Where $D_{f}$ and $D_{b}$ are the domains of definition of $f, b$ respectively and it is restricted that $(s+x, t+y) \in D_{f},(x, y) \in D_{b}$. In the definition of binary corrosion, it is required that structural elements must be completely included in the corroded set. Here min is used to replace the binary sum and multiplication is replaced with subtraction.

The computing of corrosion is to select the minimum of $f-b$ in the neighborhood determined by structural elements; so it has two kinds of effects of the corrosion operation on grayscale image:

(1) If the values of all structural elements are positive, the output image will be darker than the input one;

(2) If the bright details in the input image have smaller sizes than those of structural elements, the impact of structural elements will be weakened and the weakened degree will depend on the grayscale value surrounding these bright details as well as the shapes and magnitudes of structural elements.

\section{2) Grayscale expansion}

Conduct grayscale expansion on input image $f$ with structural element $b$ and mark it as $f \oplus b$. It is defined as follows:

$$
\begin{aligned}
& (f \oplus b)(s, t)=\max \{f(s-x, t-y)+b(x, y) \mid \\
& \left.(s-x, t-y) \in D_{f},(x, y) \in D_{b}\right\}
\end{aligned}
$$

Where $D_{f}$ and $D_{b}$ are the domains of definition of $f, b$ respectively and it is restricted that $(s-x, t-y) \in D_{f}$. As in the definition of binary expansion, it also requires in 2 operation sets, there must be at least 1 non-zero element intersected. This formula is similar to the $2 \mathrm{D}$ convolutional operation and the difference is that here it uses max in the place of the sum in convolution and the addition in the place of convolutional multiplication.

The computation of expansion is to select the maximum of $f+b$ in the neighborhood determined by structural elements; therefore, it has the following 2 effects:

(1) If all structural elements have positive values, the output image will be brighter than the input one;

(2) According to the grayscale value of the dark details in the input image as well as their shape and relationship with structural elements, they will be subtracted or removed in the expansion.

Both corrosion and expansion are irreversible. Corrosion-first-and-then-expansion usually cannot restore the signals, but generate a new morphological transform called open operation. The opposite of open operation is close operation, i.e. expansion first, corrosion later.

The expressions of open and close operation in grayscale mathematical morphology are consistent with those in binary mathematical morphology. open $f$ with $b$ (grayscale) and mark it as $f \circ b$ and its definition is as below:

$$
f \circ b=(f \Theta b) \oplus b
$$

Close $f$ with $b$ (grayscale) and mark it as $f \bullet b$. It is defined as follows:

$$
f \bullet b=(f \oplus b) \Theta b
$$

In the actual image denoising, it usually uses open operation to remove the bright details with smaller sizes than structural elements while preventing the entire grayscale value of the image and the large bright areas from being affected. On the other hand, close operation is used to eliminate the dark 
elements of small sizes compared with structural elements and it can keep the entire grayscale value of the image and the large dark areas from any effect.

In mathematical morphological image denoising process, proper selection of shapes and dimensions of structural element can improve the effect of filter denoising. In the cascade process of multi-structural element, the shapes and dimensions of structural element shall be taken into consideration. Assume that the structural element set is $A_{n m}, n$ represents the shape sequence and $m$ represents the dimension sequence, then

$A_{n m}=\left\{A_{11}, A_{12}, \cdots, A_{1 m}, A_{21}, \cdots, A_{n m}\right\}$

Where,

$A_{11} \subset A_{12} \subset \cdots \subset A_{1 m}$

$A_{21} \subset A_{22} \subset \cdots \subset A_{2 m}$

$\cdots$

$A_{n 1} \subset A_{n 2} \subset \cdots \subset A_{n m}$

Assume that morphological corrosion operation is conducted on the image, according to the aforementioned corrosion operation formula, the process is equivalent to explore and mark the position of matching structural element. If the structural elements of the same dimensions but different shapes are used to conduct morphological corrosion operation on the image, their matching times are usually different. Generally, if the structural element selected can detect such information as image edge, there are more times to be matched; otherwise, there are fewer times. Therefore, to combine the detection matching principle of structural element in the morphological corrosion process can calculate the adaptive weight according to the matching times of these elements in the image.

Assume that the weights of the structural elements of $n$ shapes are respectively: $\alpha_{1}, \alpha_{2}, \cdots, \alpha_{n}$, and the matching times in the image corrosion are $\beta_{1}, \beta_{2}, \cdots, \beta_{n}$, then the formula to calculate adaptive weight are as follows:

$$
\begin{aligned}
& \alpha_{1}=\frac{\beta_{1}}{\beta_{1}+\beta_{2}+\cdots+\beta_{n}} \\
& \alpha_{2}=\frac{\beta_{2}}{\beta_{1}+\beta_{2}+\cdots+\beta_{n}} \\
& \cdots \\
& \alpha_{n}=\frac{\beta_{n}}{\beta_{1}+\beta_{2}+\cdots+\beta_{n}}
\end{aligned}
$$

When conducting mathematical morphological filter denoising on digital image, structural elements can be adopted with dimensions from small to big in the processing according to the characteristics of noises so as to filter different noises. The use of multi-structural element in mathematical morphology can preserve as many geometrical features of digital image as possible. Therefore, series filter is selected to construct for image filtering; in another word, it filters the image with the structural element of the same shape according to the dimensions from small to big, which resembles the design procedure of series circuit. The series filter constructed by structural elements of different shapes can be connected in parallel so as to construct series-combined-parallel compound filter by integrating adaptive weight algorithm.

Assume that the input image is $f(x)$, and the string-shape filtering result after certain shape of structural element is $f_{i}(x)$ , $i=1,2, \cdots, n$, then the output image is $F(x)$. Here, the structural element determines the weights $\alpha_{1}, \alpha_{2}, \cdots, \alpha_{n}$ according to the adaptive algorithm shown in Formula (16) and then

$$
F(x)=\sum_{i=1}^{n} \alpha_{i} f_{i}(x)
$$

To simplify the experiment steps, the difference value between the result after series processing and the original image can be calculated and taken as the weight vector in specific implementation and they can be calculated through weighted sum of the series result. So, this paper has made the simulation of filtering denoising with mathematical morphological filter on digital image.

\section{TEST EXPERIMENT AND ANALYSIS}

\section{A. Image Quality Evaluation Criteria}

Image denoising is to remove noises in images with certain methods. Now there are two mainstream evaluation methods: one is subjective evaluation method and the other is to make objective evaluation according to objective image quality evaluation standards. Therefore, image quality evaluation has significant applications in image processing fields such as image denoising, image coding and image compression.

Subjective evaluation method is for human eyes to directly observe whether the image is distorted. In subjective assessment, one method is the present the finished image to a group of observers, who will decide whether the image has bright colors and clear levels and comfortable visual perception before they grade the image. In the end, lots of grade data will be processed by using the data processing methods in mathematical statistics theory to get the average grade of image quality and then the subjective image quality.

Objective evaluation method is to evaluate the image with common image quality evaluation criteria, which mainly include the following:

(1) Peak signal-to-noise ratio (PSNR)

The so-called PSNR is the peak signal of noise ratio and it is usually used to weigh the quality of processed signal. PSNR is the most common and the most widely applied objective evaluation method of image quality. It is defined as follows:

Assume that the image resolution is $M * N$ and then the PSNR is as follows

$$
\operatorname{PSNR}(d b)=10 \lg \frac{255^{2} * M * N}{\sum_{x=0}^{M-1} \sum_{y=0}^{N-1}\left[f^{\prime}(x, y)-f(x, y)\right]^{2}}
$$


Where $f(x, y)$ is the value of pixels in the original image and $f^{\prime}(x, y)$ is that in the denoised and rebuilt image. The bigger the denoised PSNR, the better the denoising effect.

(2) Signal-to-noise ratio (SNR)

In a narrow sense, SNR refers to the ratio of the output signal voltage of amplifier to the output noise voltage and it is usually represented with $\mathrm{Db}$. Image SNR should be equivalent to the ratio of power spectrum of signal to noise. But as it is difficult to calculate the power spectrum, it usually takes the approximation of ratio of variance of signal and noise and its definition is as follows:

$$
\begin{gathered}
\operatorname{SNR}(d b)=10 \lg \left(\text { power }_{\text {signal }} / \text { power }_{\text {noise }}\right) \\
\text { power }_{\text {signal }}=\frac{\sum_{x=1}^{M} \sum_{y=1}^{N} f^{2}(x, y)}{M^{*} N} \\
\text { power }_{\text {noise }}=\frac{\sum_{x=1}^{M} \sum_{y=1}^{N}\left[f(x, y)-f^{\prime}(x, y)\right]^{2}}{M^{*} N}
\end{gathered}
$$

Where $f(x, y)$ is the value of pixels in the original image and $f^{\prime}(x, y)$ is that in the denoised reconstructed image, power $_{\text {signal }}$ is the power of actual image and power ${ }_{\text {noise }}$ is the noise power. After denoising, the bigger SNR, the better the denoising effect.

\section{B. Experiment Result and Analysis}

Morphological filters are composed of open and close operations based on set theory. They don't blur image edges and they process the image with morphological operations to constitute mathematically morphological filters. This experiment select 2 different images, with Possion noise added to them. This paper conducts filter denoising experiment by constructing different filters of series and parallel filters and shows the denoising effect by computing and plotting PSNR curve, as shown in Fig.2-Fig.5 below.

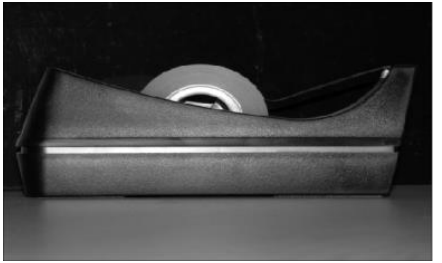

(a) Original image

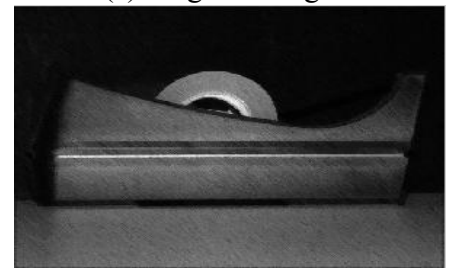

(c) Denoising results of series connection 1

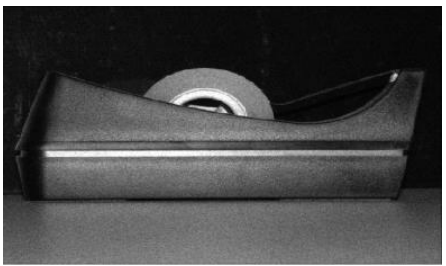

(b) Noise image

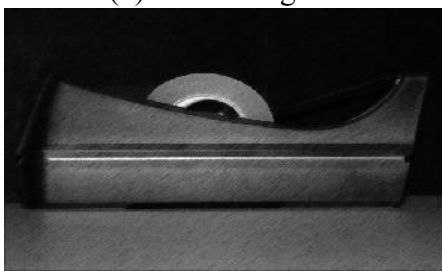

(d) Denoising results of series connection 2

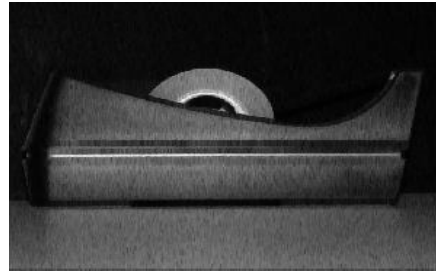

(e) Denoising results of series connection 3

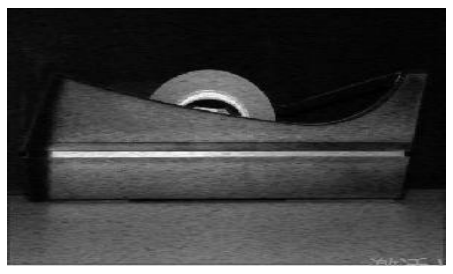

(f) Denoising results of series connection 4
Fig.2 Denoising results of series connection

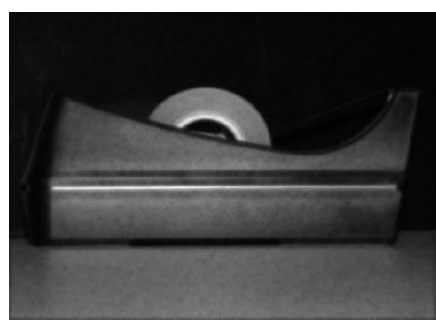

(a) Denoising results of parallel connection

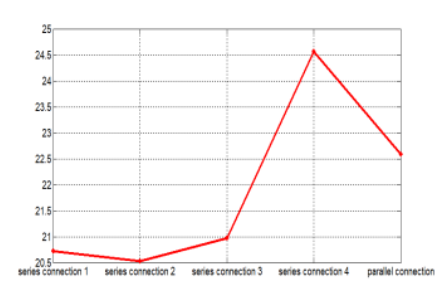

(b) Comparison of PSNR curves
Fig.3 Comparison of PSNR curves

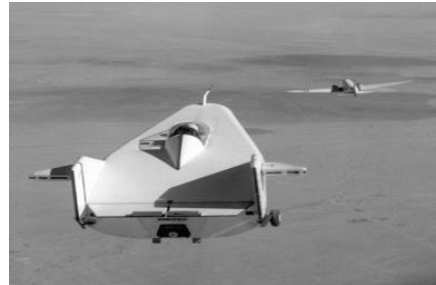

(a)Original image

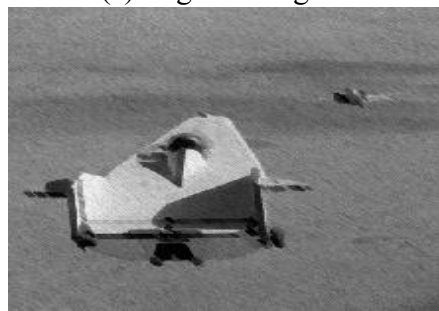

(c) Denoising results of series connection 1

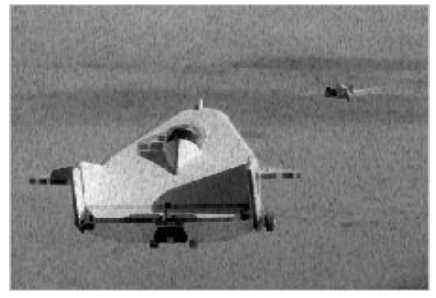

(e) Denoising results of series connection 3

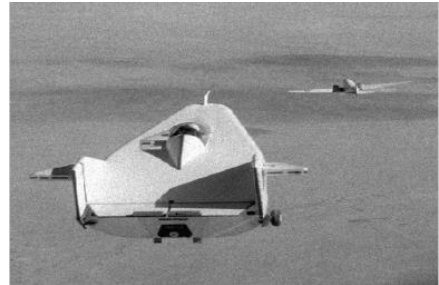

(b) Noise image

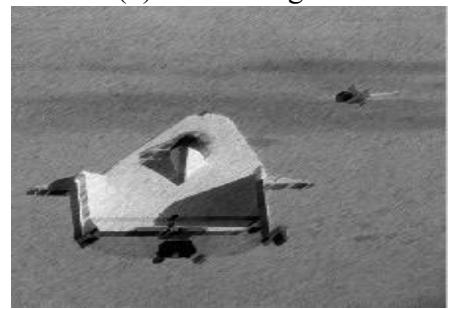

(d) Denoising results of series connection 2

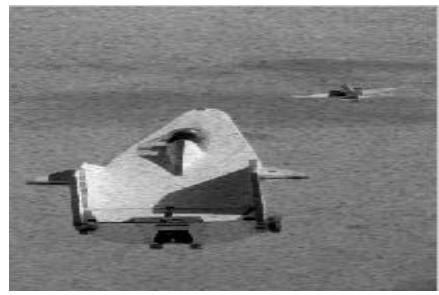

(f) Denoising results of series connection 4
Fig.4 Denoising results of series connection 


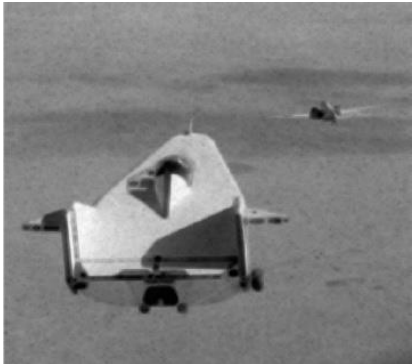

(a) Denoising results of parallel connection

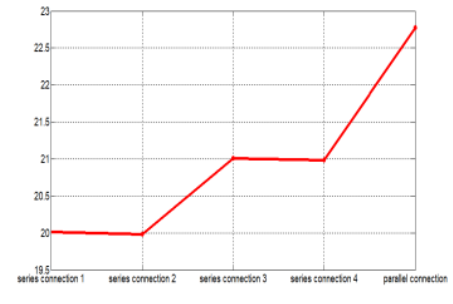

(b) Comparison of PSNR curves
Fig.5 Comparison of PSNR curves

The combination of open and close operations can be used to remove noises. Firstly, conduct open operation on the noisy image. The structural elements with larger size than noise can be selected to eliminate the image noises. So it can be learnt that this method is applicable to the images with objects of large sizes and with no tiny details and that it has excellent denoising effect on this kind of images. According to the correlation theory of set science, open and close operations are idempotent, which means that one filter can filter and remove all matching noises with structural element. The experiment result has shown that to filter with series connection usually has certain restrictions and the image also preserves relatively obvious noises; but to filter with parallel filter can have a higher PSNR value and the result is more ideal in visual effect than that with series filter only. To adopt parallel connection in filter cascade can perform morphological filtering and denoising on noisy image and lead to significant effects. It can improve to a certain extent, the effect of filter denoising through morphological filter by constructing cascade filter through series and parallel connection and applying to different image processing.

\section{CONCLUSION AND FUTURE WORK}

This paper has analyzed the generation process and characteristics of common image noises, studied the basic theories of mathematical morphology and its applications in image processing, discussed the methods to choose structural elements in mathematical morphology, proposed a filtering algorithm that has combined image denoising and mathematical morphology based on the selection of multi-structural elements in morphology, studied multi-scale structural elements and all-round structural elements, investigated the selection principles of multi-structural elements, and design the compound morphological filter with series and parallel connection. The experiment result has shown that in both visual effects and PSNR value, the method in this paper can make good results and it has led to impressive denoising effects especially for the images with rich texture details.

The future work of this paper is as follows:

(1) The selection of structural elements in this paper is based on continuous experiments, how to use some methods to automatically select the structural elements needed by the image needs further research.
(2) The adaptive filtering of morphological filter and its application in image processing are further studied.

(3) In depth study of mathematical morphology theory, let it combine with other intelligent algorithms (such as genetic algorithm, particle swarm optimization algorithm, etc.) to achieve complementary advantages and disadvantages, to improve its denoising performance.

(4) It is very important to select the appropriate structure element according to the characteristics of data. We can do further work in improving the operation efficiency and fast algorithm of morphological transformation. We can improve the existing basic morphological operation or propose a new transformation form. Some other relevant studies can be found in [27], [28], [29].

\section{REFERENCE}

[1] Ghosh, Swarup Kr; Biswas, Biswajit; Ghosh, Anupam, "SDCA: A Novel Stack Deep Convolutional Autoencoder - An Application on Retinal Image Denoising”, IET Image Processing, vol. 13, no. 14, pp.2778-2789, 2019.

[2] Bouchet, Agustina; Montes, Susana; Ballarin, Virginia, "Intuitionistic Fuzzy Set And Fuzzy Mathematical Morphology Applied To Color Leukocytes Segmentation”, Signal Image and Video Processing, vol. 14, no. 3, pp.557-564, 2020.

[3] Liu, Yepeng; Li, Xuemei; Guo, Qiang, "Adaptive Iterative Global Image Denoising Method Based on SVD", IET Image Processing, vol. 14, no. 13, pp. 3028-3038,2020.

[4] Bhujle, Hemalata; Vadavadagi, Basavaraj H.; Galaveen, Shivanand, "Efficient Non-Local Means DenoisingFor Image Sequences With Dimensionality Reduction", Multimedia Tools And Applications, vol. 77, no. 23, pp.30595-30613, 2018.

[5] Ramonaite, Rima; Petrolis, Robertas; Unay, Simge, "Mathematical Morphology-Based Imaging of Gastrointestinal Cancer Cell Motility and 5-Aminolevulinic Acid-Induced Fluorescence", Biomedical Engineering-Biomedizinische Technik, vol. 64, no. 6, pp. 711-720, 2019.

[6] Guillen-Garcia, Emmanuel; Morales-Velazquez, Luis; Luis Zorita-Lamadrid, Angel, "Accurate Identification and Characterisation of Transient Phenomena Using Wavelet Transform And Mathematical Morphology", IET Generation Transmission \& Distribution, vol. 13, no.18, pp.4021-4028, 2019.

[7] Suresh, Shilpa; Lal, Shyam, "Two-Dimensional CS Adaptive FIR Wiener Filtering Algorithm for the Denoising of Satellite Images", IEEE Journal of Selected Topics in Applied Earth Observations and Remote Sensing, vol. 10, no. 12, pp.5245-5257, 2017.

[8] Landini, Gabriel; Galton, Antony; Randell, David, "Novel Applications of Discrete Mereotopologyto Mathematical Morphology", Signal Processing-Image Communication, vol. 76, pp.109-117, 2019.

[9] Cohen, Assaf; Cohen, Aviad; Nissim, Nir, "Convolutional DenoisingAutoencoderand Siamese Networks", Neural Networks, vol. 131, pp. 64-77, 2020.

[10] Aravindan, T. E.; Seshasayanan, R, "Denoising Brain Images with the Aid of Discrete Wavelet Transform and Monarch Butterfly Optimization with Different Noises", Journal of Medical Systems, vol. 42, no. 11, 2018.

[11] Tian, Chunwei; Fei, Lunke; Zheng, Wenxian, "Deep Learning on Image Denoising: An Overview", Neural Networks, vol. 131, pp.251-275, 2020.

[12] Rezaei, Sahar; Ghafarian, Pardis; Jha, Abhinav K, "Joint Compensation of Motion and Partial Volume Effects By Iterative Deconvolution Incorporating Wavelet-Based Denoisingin Oncologic PET/CT Imaging", Physica Medica-European Journal of Medical Physics, vol. 68, pp.52-60, 2019.

[13] Sudeep, P., V; Palanisamy, R.; Kesavadas, Chandrasekharan, "An Improved Nonlocal Maximum Likelihood Estimation Method for Denoising Magnetic Resonance Images With Spatially Varying Noise Levels", Pattern Recognition Letters, vol. 139, pp.34-41, 2020. 
[14] Mafi, Mehdi; Tabarestani, Solale; Cabrerizo, Mercedes, "Denoising of Ultrasound Images Affected By Combined Speckle And Gaussian Noise", IET Image Processing, vol. 12, no.12, pp.2346-2351, 2018.

[15] Baravdish, G.; Svensson, O.; Gulliksson, M, "Damped Second Order Flow Applied to Image Denoising", IMA Journal Of Applied Mathematics, vol. 84, no. 6, pp. 1082-1111, 2019.

[16] Honzatko, David; Krulis, Martin, "Accelerating Block-Matching and 3D Filtering Method for Image Denoising on GPUs", Journal Of Real-Time Image Processing, vol. 16, no. 6, pp.2273-2287, 2019.

[17] Saravani, Shahram; Shad, Rouzbeh; Ghaemi, Marjan, "Iterative Adaptive Despeckling SAR Image Using Anisotropic Diffusion Filter and Bayesian Estimation Denoising In Wavelet Domain", Multimedia Tools And Applications, vol. 77, no. 23, pp. 31469-31486, 2018.

[18] Ali, Mohammed Nabih, "A Wavelet-Based Method for MRI Liver Image Denoising", Biomedical Engineering-Biomedizinische Technik, vol. 64, no. 6, pp.699-709, 2019.

[19] Hamid, Lydia Binti Abdul; Rosli, NennyRuthfalydia; Khairuddin, AnisSalwaMohd, "Denoising Module for Wood Texture Images", Wood Science And Technology, vol. 52, no. 6, pp.1539-1554, 2018.

[20] Brifman, Alon; Romano, Yaniv; Elad, Michael, "Unified Single-Image and Video Super-Resolution via Denoising Algorithms", IEEE Transactions on Image Processing : A Publication of the IEEE Signal Processing Society, vol. 28, no. 12, pp.6063-6076, 2019.

[21] Kittisuwan, Pichid, "Low-Complexity Image Denoising Based on Mixture Model And Simple Form of MMSE Estimation”, International Journal of Wavelets Multiresolution and Information Processing, vol. 16, no. 6, 2018.

[22] Raslain, Safia; Hachouf, Fella; Kharfouchi, Soumia, "Using A Generalised Method of Moment Approach and 2D-Generalised Autoregressive Conditional Heteroscedasticity Modelling for Denoising Ultrasound Images", IET Image Processing, vol. 12, no. 11, pp.2011-2022, 2018.

[23] Shukla, Urvashi Prakash; Nanda, SatyasaiJagannath, "Denoising Hyperspectral Images Using Hilbert Vibration Decomposition With Cluster Validation", IET Image Processing, vol. 12, no. 10, pp.1736-1745, 2018.

[24] Green, Michael; Marom, Edith M.; Konen, Eli, "Patient-Specific Image Denoisingfor Ultra-Low-Dose CT-Guided Lung Biopsies", International Journal of Computer Assisted Radiology And Surgery, vol. 12, no. 12, pp.2145-2155, 2017.

[25] Sperl, Jonathan I.; Sprenger, Tim; Tan, Ek T., "Model-Based Denoisingin Diffusion-Weighted Imaging Using Generalized Spherical Deconvolution", Magnetic Resonance in Medicine, vol. 78, no. 6, pp.2428-2438, 2017.

[26] Das, Jayanta Kumar; Choudhury, Pabitra Pal; Chaturvedi, Neelambuj, "Ranking and Clustering of Drosophila Olfactory Receptors Using Mathematical Morphology", Genomics, vol. 111, no. 4, pp. 549-559, 2019.

[27] Nur Aqilah Othman, Hamzah Ahmad, "The Analysis of Covariance Matrix for Kalman Filter based SLAM with Intermittent Measurement", Int. J. of Applied Mathematics, Computational Science and Systems Engineering, pp. 66-70, Volume 1, 2019

[28] Bacha Sawssen, Taouali Okba, Liouane Noureeddine, "A Mammographic Images Classification Technique via the Gaussian Radial Basis Kernel ELM and KPCA, pp. 92-98, Volume 2, 2020

[29] Maria Isabel Garcia-Planas, Sonia Tarragona, "Analysis of behavior of a simple eigenvalue of singular system", Int. J. of Applied Mathematics, Computational Science and Systems Engineering, pp. 41-47, Volume 3, 2021

\section{Creative Commons Attribution License 4.0 (Attribution 4.0 International, CC BY 4.0)}

This article is published under the terms of the Creative Commons Attribution License 4.0 https://creativecommons.org/licenses/by/4.0/deed.en_US 